\title{
Factores asociados con el parto prematuro entre 22 y 34 semanas en un hospital público de Santiago
}

\author{
ALFREDO OVALLE ${ }^{1}$, ELENA KAKARIEKA ${ }^{2}$, \\ GUSTAVO RENCORET ${ }^{1}$, ARIEL FUENTES ${ }^{1}$, MARÍA JOSÉ DEL RÍO ${ }^{1}$, \\ CARLA MORONG ${ }^{2}$, PABLO BENÍTEZ
}

\section{Risk factors for preterm deliveries in a public hospital}

Background: Preterm births are responsible for 75 to $80 \%$ of perinatal mortality. Aim: To determine the factors associated with preterm births, using maternal clinical data, laboratory results and pathological placental findings. Patients and Methods: Retrospective study of 642 preterm single births at 22-34 weeks' gestation. Four hundred and seven cases with pathological placental studies were included. Births were subdivided into preterm births as a consequence of a medical indication and spontaneous births with or without premature rupture of membranes (PROM). Risk factors for preterm births were classified as maternal, fetal, placental, indeterminable and unclassifiable. Results: The proportions of preterm births were spontaneous 69\% (with PROM 27\% and with intact membranes 42\%) and medically indicated births 31\%. A risk factor associated with prematurity was identified in 98 and $85 \%$ of medically indicated and spontaneous births, respectively. Ascending bacterial infection (ABI) was the most frequently associated factor with spontaneous preterm delivery in $51 \%$ of women $(142 / 280, p<0.01)$ and with preterm births of less than 30 weeks in $52 \%$ of women $(82 / 157, p<0.01)$. Vaginal or urinary infection with Group B Streptococcus, was the most common clinical condition associated with ABI related deliveries. Hypertension was present in 94 of 127 medically indicated preterm deliveries (preeclampsia in $62 \%$ and chronic hypertension in 12\%), and in 29\% (preeclampsia 24\%) of preterm births of more than 30 weeks. Congenital anomalies were mainly associated with a maternal age over 35 years in 15\% (14/92) of women. The frequency of placental diseases was higher in spontaneous preterm deliveries (14\%) and in pregnancies of more than 30 weeks in (14\%). Conclusions: $A B I$ was the most common factor associated with spontaneous preterm births at 2234 weeks, while preeclampsia is the most common factor associated with medically indicated preterm births.

(Rev Med Chile 2012; 140: 19-29).

Key words: Delivery, obstetric; Placenta; Premature birth; Vaginosis, bacterial.

'Servicio y Departamento
de Obstetricia, Ginecología
y Neonatología, Hospital
San Borja Arriarán. Facultad
de Medicina, Universidad
de Chile.
'2Servicio de Anatomía
Patológica, Hospital San
Borja Arriarán.
aAlumno de Medicina.
Recibido el 21 de marzo de
2011, aceptado el 14 de
octubre de 2011.
Correspondencia a:
Dr. Alfredo Ovalle Salas
Servicio de Obstetricia,
Ginecología y Neonatología
Hospital San Borja Arriarán
Santa Rosa 1234
Santiago, Chile.
Fono: 5555535
Fax: 2019661 - 2242410
E-mail: alfredoovalle@
gmail.com

L a frecuencia del parto prematuro en Estados Unidos de Norteamérica (USA) es entre 12 y $13 \%$ y en los países europeos entre 5 y $9 \%{ }^{1,2}$. Sin embargo, esta tasa ha crecido por incremento de los partos indicados médicamente (especialmente pre-eclampsia) y de los partos con embarazos múltiples concebidos artificialmente $\mathrm{e}^{1,3}$.
En otros lugares con menor desarrollo han aumentados los nacimientos espontáneos producto de partos prematuros con o sin rotura prematura de membranas (RPM), por incremento de la infección bacteriana ascendente (IBA) y de nuevos factores asociados detectados ${ }^{1,3}$. Este aumento ocurre a pesar de los avances en el conocimiento 
de los factores de riesgo y de la introducción de intervenciones médicas destinadas a reducir el nacimiento prematuro ${ }^{1,3}$. Además, tanto el parto prematuro espontáneo como el indicado tienden a recurrir por la misma causa ${ }^{3}$.

Los nacimientos prematuros son responsables de 75 a $80 \%$ de la mortalidad perinatal $(40 \%$ de estas muertes ocurren en los nacimientos menores de 32 semanas), de más de la cuarta parte de la morbilidad a largo plazo y de la mayoría de las complicaciones neurológicas y respiratorias que pueden presentar los neonatos ${ }^{1,3}$.

En Chile, el Ministerio de Salud en su informe técnico del año 2008, informa una incidencia de $0,99 \%$ de recién nacidos vivos menores de 32 semanas en el período 2000-20044. En el Hospital Clínico San Borja Arriarán la tasa de prematurez entre 2005 y 2007 fue 7\% (informe anual del servicio, datos no publicados).

La frecuencia de la infección ascendente es muy alta entre las 20 y 32 semanas de gestación según algunos autores. Es factor de riesgo de nacimiento espontáneo, $90 \%$ a las 24 semanas y $60 \%$ a las 32 semanas ${ }^{5,6}$. En estos eventos las lesiones inflamatorias agudas corioamnionitis y funisitis son muy frecuentes ${ }^{5-12}$.

El parto prematuro indicado ocurre por interrupción obligada de la gestación ante enfermedad materna, fetal u ovular que ponen en riesgo la salud del binomio madre-niño. En estos casos los datos clínicos maternos y de laboratorio suelen ser suficientes para diagnosticar el factor de riesgo, ya que las lesiones placentarias habitualmente inespecíficas y comunes a varias enfermedades maternas y placentarias, tienen limitado aporte al diagnóstico ${ }^{13-24}$. Mientras que en los partos espontáneos, como los datos clínicos y de laboratorio suelen ser imprecisos, el estudio placentario es determinante en la identificación del factor asociado por la presencia de marcadores histológicos específicos como corioamnionitis aguda, funisitis aguda, hallazgos propios de la IBA ${ }^{5-12}$.

El conocimiento de las condiciones asociadas con el parto prematuro permitirá establecer conductas e intervenciones que probablemente lograrán reducir el nacimiento prematuro, su recurrencia y la morbimortalidad asociada ${ }^{1,3,22}$.

El objetivo de este estudio fue conocer los factores asociados con el parto prematuro entre 22 y 34 semanas de gestación en embarazos únicos, mediante los antecedentes clínicos maternos, de laboratorio y los hallazgos histopatológicos placentarios.

La morbimortalidad perinatal de esta serie de parto prematuro será publicada en un próximo artículo.

\section{Material y Métodos}

Estudio retrospectivo de los partos prematuros entre las 22 y 34 semanas de gestación con recién nacidos únicos, vivos o muertos, que tuvieron estudio histopatológico placentario y atendidos en el Servicio de Obstetricia y Ginecología del Hospital San Borja Arriarán, en el período entre el 1 de enero de 2007 y el 30 de junio de 2009. El Comité de Etica aprobó la realización de este trabajo.

La edad gestacional se determinó por historia menstrual segura o por biometría fetal ultrasonográfica previa al ingreso. Se corroboró con las medidas antropométricas del recién nacido.

Los precursores de los partos prematuros fueron: a) partos espontáneos, con rotura prematura de membranas o con membranas intactas y b) partos indicados médicamente por patología materna, fetal u ovular ${ }^{1}$.

Según la edad materna las embarazadas se dividieron: a) menor de 20 años (adolescentes); b) entre 20 y 34 años y c) mayores de 35 años (gestantes tardías). Según la edad gestacional al parto prematuro, se establecieron dos grupos: 22,0 a 29,6 y 30,0 a 34,0 semanas de gestación.

\section{Factores asociados con el parto prematuro}

Los factores asociados se clasificaron luego de analizar en conjunto los antecedentes clínicos y de laboratorio maternos con los hallazgos histopatológicos placentarios y las diferentes propuestas aparecidas en la literatura ${ }^{7-23}$. El estudio anátomopatológico fue realizado por dos patólogos (EK y CM). Con dos o más factores presentes se seleccionó aquel de mayor importancia asociado con el parto prematuro. En estos casos la biopsia placentaria fue de utilidad para determinar el factor más relevante.

Se encontraron los siguientes factores asociados con parto prematuro:

\section{1) Maternos}

a) Infección bacteriana ascendente. Embarazo con algunas de las siguientes condiciones clínicas 
presentes sugerentes de infección intrauterina: RPM, corioamnionitis clínica, sangrado vaginal con desprendimiento amniocorial, infección cérvicovaginal (ICV) especialmente por Streptococcus Grupo B (SGB), infección del tracto urinario (ITU), dispositivo intrauterino (DIU) no extraído, placenta previa con sangrado vaginal, cérvix menor de $15 \mathrm{~mm}$ medido por ultrasonografía, desprendimiento prematuro de placenta normoinserta (DPPNI), membranas prolapsadas bajo el orificio cervical externo $(\mathrm{MPr})^{25-32}$ y asociado con los siguientes marcadores histopatológicos placentarios específicos: corioamnionitis aguda, funisitis aguda $a^{5-12}$.

\section{b) Enfermedades maternas}

Hipertensión arterial. Embarazo con hipertensión materna (preeclampsia, hipertensión arterial crónica), con algunas de las siguientes condiciones clínicas presentes: DPPNI, síndrome de HELLP, restricción del crecimiento intrauterino (RCIU), asociado con hallazgos histopatológicos placentarios inespecíficos: infarto vellositario, aterosis arterias espiraladas deciduales, hematoma retroplacentario, hemorragia intervellositaria, hemorragia subcorial, endarteritis obliterante de los vasos fetales, vasculopatía fetal trombótica y trombosis intervellositaria ${ }^{11,19,22,23}$.

Diabetes. Mellitus o gestacional, con o sin DPPNI y con o sin RCIU, asociada con hallazgos histopatológicos placentarios inespecíficos: edema vellositario, inmadurez vellositaria, maduración vellositaria retardada, infarto vellositario, hematoma retroplacentario, hemorragia subcoriónica y corangiosis ${ }^{19,22}$.

Trombofilia. Embarazada con cuadro clínico de trombosis (de extremidades o tromboembolismo pulmonar), anticuerpos antifosfolípidos positivos, con DPPNI, con o sin RCIU y con hallazgos histopatológicos placentarios inespecíficos: hemorragia y hematoma retroplacentarios, infartos vellositarios, depósito de fibrina intervellositaria, vasculopatía fetal trombótica y trombosis intervellositaria ${ }^{23}$.

Colestasis intrahepática del embarazo (CIE). Embarazada con cuadro clínico compatible, ictericia clínica y de laboratorio, con pruebas hepáticas alteradas. No se reconocen lesiones placentarias específicas.

Consumo de drogas. Consumo prolongado durante el embarazo de cocaína, marihuana, tolueno, alcohol y otras, RCIU severo, con o sin DPPNI asociado con hallazgos histopatológicos placentarios inespecíficos: infarto vellositario, hemorragia y hematoma retroplacentario ${ }^{33}$.

Otras: neumonía. Cuadro clínico y radiológico compatible ${ }^{1,6,23}$; insuficiencia renal crónica: cuadro clínico y de laboratorio compatible; lupus eritematoso diseminado, cuadro clínico y de laboratorio compatible ${ }^{34}$; cardiopatía materna, cuadro clínico y de laboratorio compatible; apendicectomía, cuadro clínico, con anatomía patológica compatible ${ }^{1,23}$; pielonefritis cuadro clínico y de laboratorio compatible ${ }^{1,23}$. En estas enfermedades no hay lesiones placentarias específicas.

\section{c) Infecciones transplacentarias}

Sifilis. Infección materna confirmada con pruebas treponémicas, con o sin infección congénita, asociada con hallazgos histopatológicos placentarios específicos: aumento del tamaño y volumen placentario, vellositis crónica, eritroblastosis de vasos fetales y arteritis obliterante $e^{6,13,22}$.

Listeria monocytogenes. Infección materna febril por L. monocytogenes aislada en sangre materna y placenta, asociada a hallazgos histopatológicos placentarios específicos: vellositis y perivellositis abscedada, microabscesos en vellosidades, corioamnios y cordón umbilical ${ }^{6,13}$.

Enfermedad periodontal. Embarazada con diagnóstico odontológico y microbiológico de periodontitis generalizada y asociada con vellositis, intervellositis en los hallazgos histopatológicos placentarios ${ }^{1,35}$.

Enfermedades virales. Infección viral materna diagnosticada por serología (inmuno globulina $\mathrm{M}$ positiva), con o sin RCIU y asociada con hallazgos histopatológicos placentarios inespecíficos: vellositis, intervellositis, perivellositis crónica, corioamnionitis crónica y con o sin hidrops fetal ${ }^{6,13,22}$.

d) Parto inducido o provocado. Embarazo interrumpido clandestinamente, por uso de misoprostol, con DPPNI y con los siguientes hallazgos histopatológicos placentarios: hemorragia $y$ hematoma retroplacentario.

\section{2) Fetales. Anomalías congénitas}

Cromosómicas (malformaciones múltiples). Confirmadas por cariograma realizado en líquido amniótico o sangre fetal. Síndrome de Turner (XO), Trisomía 18, Trisomía $21^{8,16}$. 
No cromosómicas. De causa multifactorial, producto de factores ambientales, enfermedades maternas, agentes infecciosos, físicos, químicos, uso de medicamentos o de factores mecánicos durante el primer trimestre de la gestación, sin estudio cromosómico y asociadas con lesiones vellositarias sugerentes: inclusiones del trofoblasto, estroma inmaduro, trofoblasto hipoplásico, edema vellositario o calcificaciones. Habitualmente son malformaciones de un órgano o sistema: del tubo neural, cardiacos, del riñón y vía urinaria, displasias musculoesqueléticas y otros ${ }^{8,16}$.

\section{3) Ovulares}

a) Patologías placentarias. Desprendimiento prematuro placenta normoinserta idiopático (DPPNI): separación total o parcial de la placenta del útero en embarazos sin patologías materno-fetales y con manifestaciones de asfixia aguda, shock fetal ${ }^{14-20}$. Lesiones placentarias encontradas: hematoma y hemorragia retroplacentaria, infartos vellositarios, hemorragia subcorial, trombosis intervellositaria.

Patología vascular placentaria: embarazos sin patologías materno-fetales, con manifestaciones de asfixia crónica y RCIU severo, con o sin DPPNI y con lesiones placentarias inespecíficas: arteriopatía fetal trombótica, trombosis intervellositaria y arterial fetal, depósito aumentado de fibrinoide perivellositario, infartos vellositarios, hematoma y hemorragia retroplacentaria ${ }^{14-16,22}$.

Deciduitis crónica: embarazos sin patologías materno-fetales, con o sin RCIU y asociados con lesiones histopatológicas placentarias específicas: deciduitis linfoplasmocitaria, corioamnionitis, perivellositis y vellositis crónicas, trombosis intervellositaria, aumento fibrina intervellosiaria. Síndrome conocido como "coriodeciduitis crónica": se postula que se desarrolla secundario a infección de la cavidad uterina que persiste entre embarazos y que se reactiva en la siguiente gestación ${ }^{21,22}$.

Placenta previa: embarazo con placenta previa, sangrado vaginal y sin lesiones placentarias específicas $^{14-16,31}$.

b) Patología del cordón umbilical y membranas ovulares: embarazo sin patología materno-fetal y con inserción anormal de las membranas ovulares $^{14-16}$.

\section{4) Uterinas}

Embarazos sin evidentes patologías materno- fetales y con malformaciones uterinas, útero bicorne, útero bidelfo y sin lesiones placentarias ${ }^{23}$.

\section{5) No precisables}

Casos en que los antecedentes clínicos, de laboratorio y anatomopatológicos no fueron suficientes para aclarar la condición asociada al parto prematuro $^{8}$. No clasificables casos con placenta con alteraciones involutivas, cambios secundarios a maceración placentaria por muerte fetal ${ }^{8}$.

\section{Expresión de resultados}

Para conocer el factor asociado al parto prematuro, se analizaron en conjunto los antecedentes clínicos y de laboratorio maternos con los hallazgos histopatológicos placentarios. Estos factores se estudiaron en los partos prematuros espontáneos e indicados médicamente, en relación con la edad gestacional al parto y con la edad materna.

\section{Análisis estadístico de los datos}

Se usó test de $\chi^{2}$ y test exacto de Fisher en la comparación de proporciones; Test t o test de Wilcoxon en la comparación de variables continuas y análisis de varianza según correspondiese. Un valor de $\mathrm{p}<0,05$ fue considerado significativo.

\section{Resultados}

\section{Características de la población}

En el período en estudio se atendieron 14.459 nacimientos únicos, vivos o muertos. Los partos prematuros entre 22,0 y 34,0 semanas de gestación fueron $642(4,44 \%)$. Se incluyeron 407 partos prematuros $(63,4 \%)$, con nacidos vivos $84,3 \%$ (343/407) y con muertes fetales $15,7 \%$ (64/407), que tuvieron estudio histológico placentario. Se excluyeron 235 casos sin biopsia placentaria.

Según los precursores obstétricos de los nacimientos prematuros, éstos fueron espontáneos 68,8\% (280/407) [con RPM 27,0\% (110/407), con membranas intactas $41,8 \%(170 / 407)]$ e indicados médicamente $31,2 \%$ (127/407) (Figuras 1 y 2). Contribuyeron con el parto prematuro: $17,2 \%$ (70/407) las adolescentes (< 20 años), 60,2\% (245/407) las mujeres entre 20 y 34 años), y 22,6\% (92/407) las gestantes tardías (35 años y más). Los nacimientos menores de 30 semanas fueron $38,6 \%$ (157/407) y los mayores de 30 semanas $61,4 \%$ (250/407). 

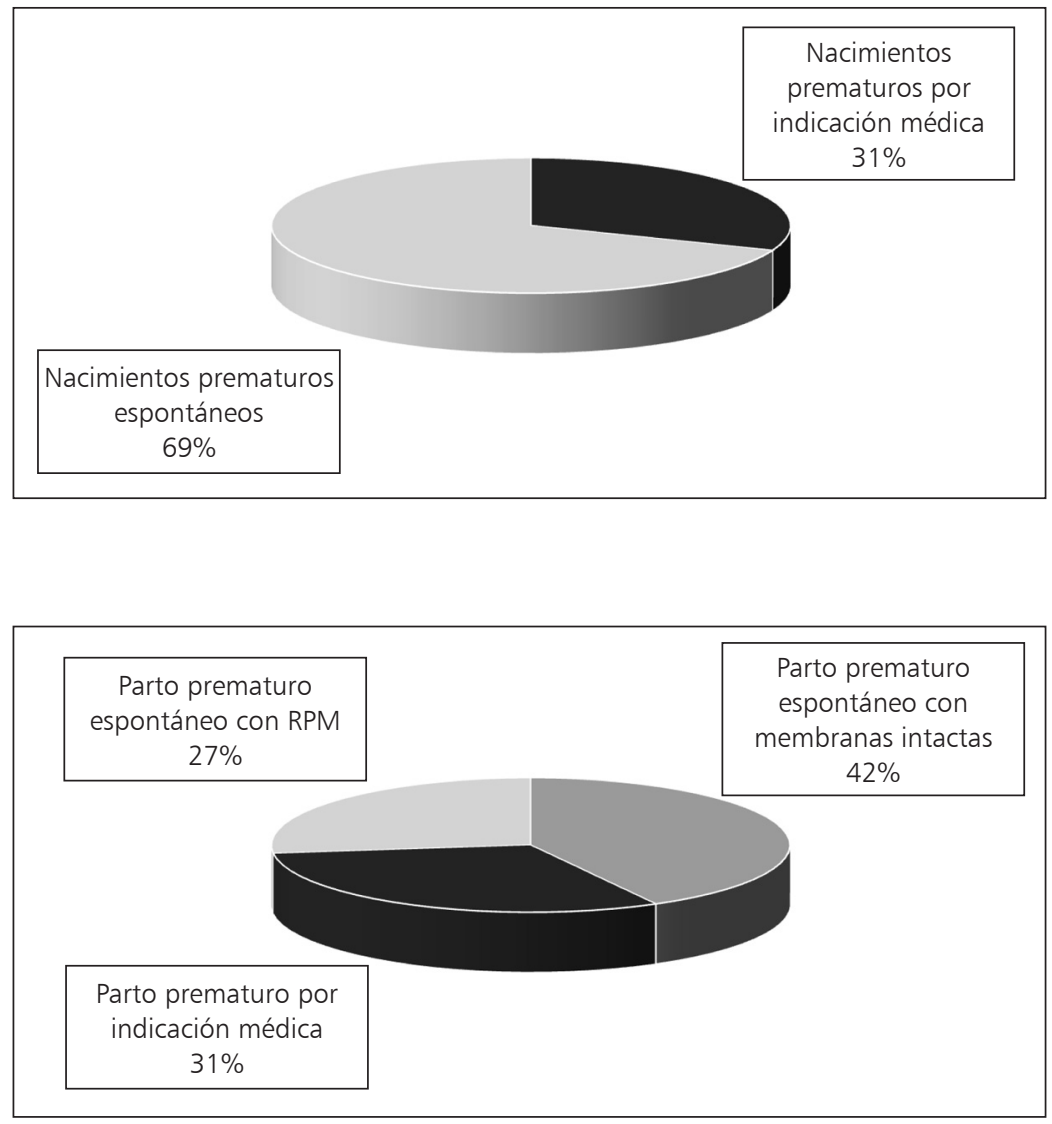

Figura 1. Nacimientos prematuros entre 22-34 semanas. Hospital San Borja Arriarán 2007-2009.
Figura 2. Precursores obstétricos del parto prematuro entre 22-34 semanas. Hospital San Borja Arriarán 2007-2009.
Características generales de la población (Tabla 1)

El parto espontáneo ocurrió a menor edad gestacional, media 29,5 vs 31,1 semanas $p<0,01$ y más frecuentemente bajo las 30 semanas $43,2 \%$ $(121 / 280)$ vs $28,3 \%(36 / 127)$ que el parto indicado $\mathrm{p}<0,01$.

\section{Factores asociados con el parto prematuro entre 22 y 34 semanas}

Se pudo conocer el factor asociado con el parto prematuro en $89,2 \%$ de los casos (363/407), indicados (98\%), espontáneos (85\%) (Tabla 2).

\section{Factores asociados según precursores obstétricos} de los nacimientos prematuros (Tabla 3 )

Se presentaron más frecuentemente, la IBA $50,7 \%(142 / 280) \mathrm{p}<0,0001$ en los nacimientos espontáneos y la hipertensión arterial 74,0\% (94/127) [preeclampsia 62,2\% (79/127)] p < 0,0001 en los partos indicados. La identificación del factor asociado a la prematurez fue mayor en los partos indicados 98,4\% (125/127) que en los espontáneos $85 \%(238 / 280) \mathrm{p}<0,001$.

\section{Factores asociados según la edad materna (Tabla 4)}

La anomalía congénita apareció más frecuentemente en la gestantes tardía 15,2\% (14/92) $(\mathrm{p}<0,03)$. La preeclampsia se presentó más frecuentemente en la mujer entre 20 y 34 años $23,6 \%(58 / 245)(\mathrm{p}<0,05)$. La hipertensión crónica apareció en la mujer sobre 35 años 9,8\% (9/92) < 0,01 . La IBA, otras enfermedades maternas, infecciones transplacentarias y patologías placentarias, se presentaron sin diferencias en las diferentes edades maternas.

\section{Factores asociados según la edad gestacional (Tabla 5)}

La IBA se presentó más frecuentemente en el 
Tabla 1. Características de la población en estudio. Parto prematuro entre 22-34 semanas. Hospital San Borja Arriarán 2007-2009

\begin{tabular}{|c|c|c|c|}
\hline & $\begin{array}{c}\text { Partos prematuros } \\
\text { espontáneos } \\
n=280\end{array}$ & $\begin{array}{c}\text { Partos prematuros } \\
\text { indicados } \\
n=127\end{array}$ & $\mathbf{P}$ \\
\hline $\begin{array}{l}\text { Edad materna (años) } \\
\text { Media (ES) } \\
\text { Rango }\end{array}$ & $\begin{array}{r}27,5(0,49) \\
13-45\end{array}$ & $\begin{array}{r}28,0(0,63) \\
17-42\end{array}$ & NS \\
\hline $\begin{array}{l}\text { Paridad } \\
\text { Primípara } \\
\text { Multípara }\end{array}$ & $\begin{array}{l}130(46,4 \%) \\
150(53,6 \%)\end{array}$ & $\begin{array}{l}66(52,0 \%) \\
61(48,0 \%)\end{array}$ & NS \\
\hline $\begin{array}{l}\text { Vía del parto } \\
\text { Vaginal } \\
\text { Cesárea }\end{array}$ & $\begin{array}{l}171(61,1 \%) \\
109(38,9 \%)\end{array}$ & $\begin{array}{r}8(6,3 \%) \\
119(93,7 \%)\end{array}$ & $<0,01$ \\
\hline $\begin{array}{l}\text { Edad gestacional parto prematuro (semanas) } \\
\text { Media (EE) } \\
\text { Rango } \\
22-29,6 \\
30-34,0\end{array}$ & $\begin{array}{r}29,5(0,21) \\
22-34 \\
121(43,2 \%) \\
159(56,8 \%)\end{array}$ & $\begin{array}{r}31.1(0.23) \\
23-34 \\
36(28.3 \%) \\
91(71.7 \%)\end{array}$ & $\begin{array}{l}<0,01 \\
<0,01\end{array}$ \\
\hline $\begin{array}{l}\text { Peso RN (gramos) } \\
\text { Media (EE) } \\
\text { Rango } \\
<750-1.499 \\
1.500-2.499 \\
2.500 \text { y más }\end{array}$ & $\begin{array}{r}1.459(34,8) \\
300-2.610 \\
141(50,4 \%) \\
138(49,3 \%) \\
1(0,3 \%)\end{array}$ & $\begin{array}{r}1.451(36,6) \\
560-2.410 \\
67(52,7 \%) \\
60(47,2 \%) \\
0\end{array}$ & $\begin{array}{l}\text { NS } \\
\text { NS } \\
\text { NS } \\
\text { NS }\end{array}$ \\
\hline
\end{tabular}

EE: Error estándar.

embarazo menor de 30 semanas 52,2\% (82/157) $\mathrm{p}<0,0001$. En cambio la preeclampsia y las patologías placentarias se presentaron más frecuentemente en los embarazos mayores de 30 semanas: $24,4 \%(61 / 250) \mathrm{p}<0,02$.

Las condiciones clínicas que más frecuentemente se presentaron en el parto prematuro por IBA (Tabla 6) fueron: a) infección génitourinaria (vaginal+urinaria) por SGB 19,4\% (13/67) (en 7 casos ICV única patología, en 4 ICV asociada con una de las siguientes situaciones: sangrado vaginaldesprendimiento amniocorial, ITU, DPPNI, DIU no extraído y en 2 asociada con diabetes; la edad gestacional promedio del parto prematuro asociada con infección génitourinaria por SGB fue 27 semanas); para el diagnóstico de SGB en muestra vaginal se usó cultivo en medio no selectivo; b) sangrado vaginal con desprendimiento amniocorial $19,4 \%(13 / 67)$ y c) ITU baja o bacteriuria asintomática $16,4 \%(11 / 67)$ (8 casos por Escherichia coli y un caso de cada uno por Streptococcus faecalis, Klebsiella pneumoniae y SGB).

\section{Discusión}

El parto prematuro es la principal causa de morbilidad y mortalidad perinatal en todo el mundo ${ }^{1}$ especialmente en los nacimientos menores de 34 semanas $^{1,3}$. El conocimiento de los factores de riesgo del parto prematuro temprano es muy importante por la posibilidad de impedir su recurrencia que habitualmente se produce por la misma causa ${ }^{3}$.

En este estudio los nacimientos prematuros espontáneos entre 22 y 34 semanas representaron el $69 \%$ de la serie, en tanto que los nacimientos por indicación médica fueron $31 \%$. Estas frecuencias, son similares a las descritas en nacimientos únicos prematuros menores de 37 semanas en USA ${ }^{1,3}$.

La IBA y la patología placentaria se presentaron más frecuentemente en los nacimientos espontáneos, mientras que la hipertensión arterial (preeclampsia) ocurrió más frecuentemente en los partos indicados médicamente.

La IBA además de ser el factor más frecuente en este estudio, se asoció con más de la mitad de 
Factores asociados con el parto prematuro - A. Ovalle et al

Tabla 2. Factores asociados con el parto prematuro entre 22 y 34 semanas. Hospital San Borja Arriarán 2007-2009

\begin{tabular}{|c|c|c|c|}
\hline Factores asociados & & $\mathbf{n}$ & (\%) \\
\hline \multicolumn{4}{|l|}{ Maternos } \\
\hline \multirow{10}{*}{$\begin{array}{l}\text { Infección bacteriana ascendente } \\
\text { Enfermedades maternas }\end{array}$} & & 147 & $(36,1)$ \\
\hline & Hipertensión arterial & 98 & $(24,1)$ \\
\hline & Preeclampsia & 82 & $(20,1)$ \\
\hline & Hipertensión crónica & 16 & $(4,0)$ \\
\hline & Otras enfermedades médicas & 28 & $(6,9)$ \\
\hline & Diabetes & 5 & $(1,2)$ \\
\hline & Trombofilia & 4 & $(1,0)$ \\
\hline & $\mathrm{CIE}$ & 2 & $(0,5)$ \\
\hline & Consumo drogas & 6 & $(1,5)$ \\
\hline & Otras & 11 & $(2,7)$ \\
\hline \multirow{5}{*}{ Infecciones transplacentarias } & & 11 & $(2,7)$ \\
\hline & Sífilis & 2 & $(0,5)$ \\
\hline & Lysteria monocytogenes & 2 & $(0,5)$ \\
\hline & Enfermedad periodontal & 1 & $(0,2)$ \\
\hline & Enfermedades virales (vellositis crónica inespecífica) & 6 & $(1,5)$ \\
\hline Parto inducido o provocado & & 3 & $(0,7)$ \\
\hline \multicolumn{4}{|l|}{ Fetales } \\
\hline \multirow[t]{3}{*}{ Anomalías congénitas } & & 29 & $(7,1)$ \\
\hline & Cromosómicas & 5 & $(1,2)$ \\
\hline & No cromosómicas & 24 & $(5,9)$ \\
\hline \multicolumn{4}{|l|}{ Ovulares } \\
\hline \multirow[t]{5}{*}{ Patologías placentarias } & & 45 & $(11,1)$ \\
\hline & DPPNI idiopático & 20 & $(4,9)$ \\
\hline & Patología vascular placentaria & 13 & $(3,2)$ \\
\hline & Deciduitis crónica & 10 & $(2,5)$ \\
\hline & Placenta previa & 2 & $(0,5)$ \\
\hline Otras causas & & 2 & $(0,5)$ \\
\hline Patologías de cordón - membranas & & 1 & $(0,2)$ \\
\hline \multicolumn{4}{|l|}{ Uterinos } \\
\hline Malformaciones & & 1 & $(0,2)$ \\
\hline No clasificables & & 1 & $(0,2)$ \\
\hline \multirow[t]{2}{*}{ No precisables } & & 43 & $(10,6)$ \\
\hline & & 44 & $(10,8)$ \\
\hline Total & & 407 & $100,0)$ \\
\hline
\end{tabular}

CIE, colestasis intrahepática del embarazo.

los nacimientos prematuros espontáneos, con el $71 \%$ de los casos con RPM y con el $52 \%$ de los partos menores de 30 semanas. Se manifestó clínicamente como infección vaginal y/o urinaria por SGB, sangrado vaginal con desprendimiento amniocorial del polo inferior e ITU. La infección génitourinaria por SGB (19\%) resultó la condición clínica más frecuente en los partos prematuros por esta causa y se presentó en el $2^{\circ}$ trimestre ( 27 semanas promedio). En la mitad de estos casos ocurrió muerte perinatal por neumonía congénita (dato de próxima publicación). También la IBA apareció en pacientes con sangrado vaginal-desprendimiento amniocorial, DPPNI, dispositivo intrauterino no extraído, placenta previa con sangrado vaginal, cérvix acortado, membranas prolapsadas, diabetes y malformaciones uterinas. En casi todas estas condiciones obstétricas se ha encontrado aumento del parto prematuro por incremento de infección intraamniótica ${ }^{25-32}$. Estos resultados sugieren que, posiblemente la pesquisa y tratamiento de las ICV, especialmente por SGB en el $2^{\circ}$ trimestre, el manejo oportuno con antibióticos del sangrado vaginal-desprendimiento amniocorial y de las 
Tabla 3. Factores asociados según precursores de parto prematuro. Hospital San Borja Arriarán 2007-2009

\begin{tabular}{|c|c|c|c|c|c|}
\hline Factores asociados & $\begin{array}{l}\text { Con RPM } \\
\begin{array}{l}\text { (a) } \\
n=110\end{array}\end{array}$ & $\begin{array}{c}\text { Con membranas } \\
\text { intactas } \\
\text { (b) } \\
n=170\end{array}$ & $\begin{array}{l}\text { Espontáneo } \\
\qquad \begin{array}{c}(a+b) \\
n=280\end{array}\end{array}$ & $\begin{array}{c}\text { Indicado } \\
\text { medicamente } \\
\text { (c) } \\
n=127\end{array}$ & $\begin{array}{c}p \\
(a+b) \\
v s \\
(c)\end{array}$ \\
\hline IBA & $78(70,9 \%)$ & $64(37,6 \%)$ & $142(50,7 \%)$ & $5 \quad(3,9 \%)$ & $<0,0001$ \\
\hline $\begin{array}{l}\text { Hipertensión arterial } \\
\text { Preeclampsia } \\
\text { Hipertensión crónica }\end{array}$ & $\begin{array}{l}0 \\
0 \\
0\end{array}$ & $\begin{array}{ll}4 & (2,4 \%) \\
3 & (1,8 \%) \\
1 & (0,6 \%)\end{array}$ & $\begin{array}{ll}4 & (1,4 \%) \\
3 & (1,1 \%) \\
1 & (0,3 \%)\end{array}$ & $\begin{array}{l}94(74,0 \%) \\
79(62,2 \%) \\
15(11,8 \%)\end{array}$ & $\begin{array}{l}<0,0001 \\
<0,0001 \\
<0,0001\end{array}$ \\
\hline $\begin{array}{l}\text { Otra enfermedad médica } \\
\text { materna }\end{array}$ & $7 \quad(6,4 \%)$ & $7 \quad(4,1 \%)$ & $14 \quad(5,0 \%)$ & $14(11,0 \%)$ & $<0,03$ \\
\hline Infección transplacentaria & $3(2,7 \%)$ & $6 \quad(3,5 \%)$ & $9(3,2 \%)$ & $2(1,6 \%)$ & NS \\
\hline Parto inducido & 0 & 0 & 0 & $3(2,4 \%)$ & NS \\
\hline Anomalía congénita & $3 \quad(2,7 \%)$ & $26(15,3 \%)$ & $29(10,4 \%)$ & 0 & NS \\
\hline Patología placentaria & $8(7,3 \%)$ & $30(17,6 \%)$ & $38(13,6 \%)$ & $7 \quad(5,5 \%)$ & $<0,02$ \\
\hline Otras & $2(1,8 \%)$ & 0 & $2(0,7 \%)$ & 0 & NS \\
\hline $\begin{array}{l}\text { No precisable } \\
\text { No clasificable }\end{array}$ & $9(8,2 \%)$ & $33(19,4 \%)$ & $42(15,0 \%)$ & $2(1,6 \%)$ & $<0,001$ \\
\hline
\end{tabular}

IBA: Infección bacteriana ascendente.

Tabla 4. Factores asociados con parto prematuro según edad materna. Hospital San Borja Arriarán 2007-2009

\begin{tabular}{|c|c|c|c|c|}
\hline Factores asociados & $\begin{array}{c}\text { menor de } 20 \\
\text { años } \\
\text { (a) } \\
n=70\end{array}$ & $\begin{array}{c}\text { entre } 20 \text { y } 34 \\
\text { años } \\
\text { (b) } \\
n=245\end{array}$ & $\begin{array}{c}35 \text { años y más } \\
\text { (c) } \\
n=92\end{array}$ & $\mathbf{p}$ \\
\hline IBA & $30(42,9 \%)$ & $81(33,1 \%)$ & $36(39,1 \%)$ & NS \\
\hline $\begin{array}{l}\text { Hipertensión arterial } \\
\text { Preeclampsia } \\
\text { Hipertensión crónica }\end{array}$ & $\begin{array}{l}12(17,1 \%) \\
12(17,1 \%) \\
0\end{array}$ & $\begin{array}{r}65(26,5 \%) \\
58(23,6 \%) \\
7 \quad(2,9 \%)\end{array}$ & $\begin{array}{r}21(22,8 \%) \\
12(13,0 \%) \\
9 \quad(9,8 \%)\end{array}$ & $\begin{array}{l}\text { NS } \\
<0,05(\mathrm{~b}) \text { vs (c) } \\
<0,01(\mathrm{a}+\mathrm{b}) \text { vs (c) }\end{array}$ \\
\hline Otra enfermedad médica materna & $6(8,6 \%)$ & $19(7,8 \%)$ & $3(3,3 \%)$ & NS \\
\hline Infección transplacentaria & $1 \quad(1,4 \%)$ & $5 \quad(2,0 \%)$ & $5 \quad(5,4 \%)$ & NS \\
\hline Parto inducido & 0 & $2(0,8 \%)$ & $1 \quad(1,1 \%)$ & NS \\
\hline Anomalía congénita & $2(2,9 \%)$ & $13(5,3 \%)$ & $14(15,2 \%)$ & $<0,03$ (a) vs (c) \\
\hline Patología placentaria & $10(14,3 \%)$ & $30(12,2 \%)$ & $5 \quad(5,4 \%)$ & NS \\
\hline Otras & 0 & $2(1,6 \%)$ & 0 & NS \\
\hline No precisable - No clasificable & $9(12,9 \%)$ & $28(11,4 \%)$ & $7 \quad(7,6 \%)$ & NS \\
\hline
\end{tabular}

IBA: Infección bacteriana ascendente. 
Factores asociados con el parto prematuro - A. Ovalle et al

Tabla 5. Factores asociados con parto prematuro según edad gestacional. Hospital San Borja Arriarán 2007-2009

\begin{tabular}{|c|c|c|c|}
\hline $\begin{array}{l}\text { Factores } \\
\text { asociados }\end{array}$ & $\begin{array}{c}22,0-29,6 \\
\text { semanas } \\
n=157\end{array}$ & $\begin{array}{c}30,0-34,0 \\
\text { semanas } \\
n=250\end{array}$ & $\mathbf{p}$ \\
\hline IBA & $82(52.2 \%)$ & $65(26,0 \%)$ & $<0,001$ \\
\hline $\begin{array}{l}\text { Hipertensión arterial } \\
\text { Preeclampsia } \\
\text { Hipertensión crónica }\end{array}$ & $\begin{array}{c}26(16,6 \%) \\
21(13,4 \%) \\
5(3,2 \%)\end{array}$ & $\begin{array}{c}72(28,8 \%) \\
61(24,4 \%) \\
11(4,4 \%)\end{array}$ & $\begin{array}{l}<0,01 \\
<0,01 \\
\text { NS }\end{array}$ \\
\hline $\begin{array}{l}\text { Otra enfermedad } \\
\text { médica materna }\end{array}$ & $6(3,8 \%)$ & $22(8,8 \%)$ & NS \\
\hline $\begin{array}{l}\text { Infección } \\
\text { transplacentaria }\end{array}$ & $4(2,5 \%)$ & $7(2,8 \%)$ & NS \\
\hline Parto inducido & $3(1,9 \%)$ & 0 & NS \\
\hline Anomalía congénita & $13(8,3 \%)$ & $16(6,4 \%)$ & NS \\
\hline Patología placentaria & $10(6,4 \%)$ & $35(14,0 \%)$ & $<0,02$ \\
\hline Otras & $1(0,6 \%)$ & $1(0,4 \%)$ & NS \\
\hline $\begin{array}{l}\text { No precisable } \\
\text { No clasificable }\end{array}$ & $12(7,6 \%)$ & $32(12,8 \%)$ & NS \\
\hline
\end{tabular}

IBA: Infección bacteriana ascendente.

otras condiciones clínicas presentes sugerentes de infección intrauterina, debieran ser medidas apropiadas para reducir el nacimiento prematuro espontáneo asociado con este factor de riesgo.

La hipertensión arterial (mayoritariamente preeclampsia severa) fue muy usual en los nacimientos indicados de esta serie (74\%) y entre las 30 y 34 semanas de gestación. La interrupción del embarazo fue por el elevado riesgo materno fetal. El aumento de los partos indicados en nuestro estudio ocurrió asociado con una probable declinación de la mortalidad perinatal ${ }^{3,36}$. La obesidad, no medida en este grupo, es otra de las probables razones del aumento de la hipertensión arterial encontrada ${ }^{37,38}$.

La diabetes frecuentemente asociada con hipertensión arterial e IBA apareció comúnmente en el parto indicado médicamente. La obesidad posiblemente constituyó factor de riesgo para la diabetes ${ }^{37}$ al igual que para la hipertensión arterial.

La anomalía congénita, habitualmente no cromosómica, de causa multifactorial, se presentó más frecuentemente en las gestantes tardías y como parto prematuro espontáneo. En la generalidad de las publicaciones el parto prematuro por anomalía fetal es indicado ${ }^{36,39}$.
Tabla 6. Condición clínica asociada con el parto prematuro por infección bacteriana ascendente. Hospital San Borja Arriarán 2007-2009

\begin{tabular}{|c|c|}
\hline Condición clínica asociada & $n=67 *$ \\
\hline $\begin{array}{l}\text { Sangrado vaginal-desprendimiento } \\
\text { amniocorial** }\end{array}$ & $13(19,4 \%)$ \\
\hline Infección urinaria** & $11(16,4 \%)$ \\
\hline $\begin{array}{l}\text { Desprendimiento prematuro de } \\
\text { placenta normo inserta** }\end{array}$ & $9(13,4 \%)$ \\
\hline Infección vaginal por SGB & $7(10,4 \%)$ \\
\hline Diabetes: mellitus o gestacional ${ }^{\circ}$ & $6(9,0 \%)$ \\
\hline Dispositivo intrauterino no extraído** & $6 \quad(9,0 \%)$ \\
\hline Placenta previa & $5 \quad(7,5 \%)$ \\
\hline Malformación uterina & $3(4,5 \%)$ \\
\hline Cérvix acortado & $2(3,0 \%)$ \\
\hline Membranas prolapsadas & $1(1,5 \%)$ \\
\hline Otras & $4(6,0 \%)$ \\
\hline
\end{tabular}

*80 casos sin datos clínicos consignados. SGB: Streptococcus Grupo B. ${ }^{* *} 1$ caso de cada uno con SGB vaginal. ${ }^{2} 2$ casos con SGB vaginal. Infección génitourinaria (vaginal + urinaria) por SGB 13/67 (19,4\%).

En esta casuística, como la mayoría de las malformaciones fueron complejas y graves con muerte fetal (en Chile no se permite el aborto terapéutico), el nacimiento fue espontáneo.

La patología placentaria, fundamentalmente DPPNI idiopático, deciduitis crónica y patología vascular se manifestaron preferentemente en los nacimientos prematuros espontáneos y en gestaciones mayores de 30 semanas. Este resultado, al igual que el anterior, es más frecuente por indicación médica, en la literatura ${ }^{36,39}$. La explicación puede ser, falla en diagnosticar patología placentaria y mantener conducta conservadora prolongada en gestaciones menores de 1.500 gramos con RCIU, favoreciendo el parto prematuro espontáneo.

La limitación de este estudio fue la exclusión de 235 casos sin biopsia placentaria, no obstante que los datos clínicos maternos y de laboratorio suelen ser suficientes para precisar el factor de riesgo en los partos indicados. A pesar de esto, fue necesario 
excluir estos casos, porque la biopsia placentaria fue importante para determinar el factor asociado cuando hay dos presentes. Además este error en la selección de pacientes no debió modificar los resultados porque la decisión de no estudiar la placenta fue al azar, sin existir ningún criterio específico de descarte de estas biopsias. Aun más, los datos clínicos y de laboratorio de los casos excluídos permiten sugerir que la distribución de los posibles factores asociados en este grupo no fue diferente.

\section{Referencias}

1. Goldenberg RL, Culhane JF, Iams JD, Romero R. Epidemiology and causes of preterm birth. Lancet 2008; 371: 75-84. Review.

2. Macdorman MF, Mathews TJ. BirthStats: percentage of preterm births, United States and selected European countries, 2004. Birth 2010; 37: 168.

3. Ananth CV, Vintzileos AM. Epidemiology of preterm birth and its clinical subtypes. J Matern Fetal Neonatal Med 2006; 19: 773-82. Review.

4. Morgues M, Reyes C. Informe técnico. Recién nacidos con menos de 32 semanas de edad gestacional. Sistema Nacional de Servicios de Salud de Chile. Quinquenio 2000-2004. Subsecretaria de Redes Asistenciales. Minsal. www.prematuros.cl 2008.

5. Sebire NJ, Goldin RD, Regan L. Histological chorioamnionitis in relation to clinical presentation at $14-40$ weeks of gestation. J Obstet Gynaecol 2001; 21: 242-5.

6. Al-Adnani M, Sebire NJ. The role of perinatal pathological examination in subclinical infection in obstetrics. Best Pract Res Clin Obstet Gynaecol 2007; 21: 505-21. Review.

7. Goldenberg RL, Andrews WW, Faye-Petersen O, Cliver S, Goepfert AR, Hauth JC. The Alabama Preterm Birth Project: placental histology in recurrent spontaneous and indicated preterm birth. Am J Obstet Gynecol 2006; 195: 792-6.

8. Ovalle A, Kakarieka E, Correa A, Vial MT, Aspillaga C. Estudio anátomo-clínico de las causas de muerte fetal. Rev Chil Obstet Ginecol 2005; 70: 303-12.

9. Ovalle A, Martínez MA, Kakarieka ME, Gómez R, Torres J, Fuentes A, et al. Histopatología de la placenta en la rotura prematura de membranas de pretérmino. Relación con la microbiología aislada y con los resultados maternoneonatales. Rev Med Chile 1998; 126: 930-42.

10. Ovalle A, Gómez R, Martínez MA, Kakarieka E, Fuentes A, Aspillaga C, et al. Invasión microbiana de la cavidad amniotica en la rotura de membranas de pretérmino.
Resultados maternoneonatales y patología placentaria según microorganismo aislado. Rev Med Chile 2005; 133: 51-61.

11. Arias F, Victoria A, Cho K, Kraus F. Placental histology and clinical characteristics of patients with preterm premature rupture of membranes. Obstet Gynecol 1997; 89: 265-71.

12. Park CW, Moon KC, Park JS, Jun JK, Romero R, Yoon $\mathrm{BH}$. The Involvement of Human Amnion in Histologic Chorioamnionitis is an Indicator that a Fetal and an Intra-Amniotic Inflammatory Response is More Likely and Severe: Clinical Implications Placenta 2009; 30: 5661.

13. Bernischke K, Kaufman P. Pathology of the human placenta. Chapter: Histopathological Approach to Villous Alteration p 419-434. Chapter: Infectious diseases p 591-659. New York NY: Springer Verlag NY, Inc; 2001.

14. Perrin VDK. Pathology of the Placenta. Chapter: Placenta as a Reflection of maternal disease p 57-70. New York, Churchill Livingstone Inc; 1984.

15. Fox H. Pathology of the Placenta. Chapter: Histological Abnormalities of the Placenta p 149-197. Philadelfia, Pa: WB Saunders Co; 1978.

16. Keeling JW. Fetal and Neonatal Pathology. Spriger-Verlag. Berlin Heidelgerg 1987.

17. Boog G. Chronic villitis of unknown etiology. Eur J Obstet Gynecol Reprod Biol 2008; 136: 9-15. Review.

18. Redline RW. Villitis of unknown etiology: noninfectious chronic villitis in the placenta. Hum Pathol 2007; 38: 1439-46.

19. Kovo M, Schreiber L, Ben-Haroush A, Wand S, Golan A, Bar J. Placental vascular lesion differences in pregnancyinduced hypertension and normotensive fetal growth restriction. Am J Obstet Gynecol 2010; 202: 561.e1-5.

20. Salafia CM, Ernst LM, Pezzullo JC, Wolf EJ, Rosenkrantz TS, Vintzileos AM. The very low birthweight infant: maternal complications leading to preterm birth, placental lesions, and intrauterine growth. Am J Perinatol 1995; 12: 106-10.

21. Hecht JL, Allred EN, Kliman HJ, Zambrano E, Doss BJ, Husain A, et al. Elgan Study Investigators. Histological characteristics of singleton placentas delivered before the 28th week of gestation. Pathology 2008; 40: 372-6.

22. Redline RW. Placental Pathology: A Systematic Approach with Clinical Correlations. Placenta 29, Supplement A, Trophoblast Research, Vol. 22 (2008) S86eS91.

23. Mcparland P, Jones G, Taylor D. Preterm labour and prematurity. Current Obstetrics \& Gynaecology 2004; 14: 309-19.

24. Iams JD, Romero R, Culhane JF, Goldenberg RL. Primary, secondary, and tertiary interventions to reduce the 
Factores asociados con el parto prematuro - A. Ovalle et al

morbidity and mortality of preterm birth. Lancet 2008; 371: 164-75.

25. Rezeberga D, Lazdane G, Kroica J, Sokoloval, Donders GG. Placental histological inflammation and reproductive tract infections in a low risk pregnant population in Latvia Acta Obstetricia et Gynecologica 2008; 87: 360-5.

26. Ganer H, Levy A, Ohel I, Sheiner E. Pregnancy outcome in women with an intrauterine contraceptive device. Am J Obstet Gynecol 2009; 201: 381.e1-5.

27. Sheiner E, Mazor-Drey E, Levy A. Asymptomatic bacteriuria during pregnancy. J Matern Fetal Neonatal Med 2009; 22: 423-7.

28. Håkansson S, Källén K. Impact and risk factors for earlyonset group Bstreptococcal morbidity: analysis of a national, population-based cohort in Sweden 1997-2001. BJOG 2006; 113: 1452-8.

29. Gómez R, Romero R, Nien JK, Medina L, Carstens M, Kim YM, et al. Idiopathic vaginal bleeding during pregnancy as the only clinical manifestation of intrauterine infection. J Matern Fetal Neonatal Med 2005; 18: 31-7.

30. Álvarez JR, Fechner AJ, Williams SF, Ganesh VL, Apuzzio JJ. Asymptomatic bacteriuria in pregestational diabetic pregnancies and the role of group B Streptococcus. Am J Perinatol 2010; 27: 231-4.

31. Madan I, Romero R, Kusanovic JP, Mittal P, Chaiworapongsa $T$, Dong $Z$, et al. The frequency and clinical significance of intra-amniotic infection and/or inflammation in women with placenta previa and vaginal bleeding: an unexpected observation. J Perinat Med 2010; 38: 275-9.
32. Hassan S, Romero R, Hendler I, Gómez R, Khalek N, Espinoza J, et al. A sonographic short cérvix as the only clinical manifestation of intra-amniotic infection. J Perinat Med 2006; 34: 13-9.

33. Pinto SM, Dodd S, Walkinshaw SA, Siney C, Kakkar P, Mousa HA. Substance abuse during pregnancy: effect on pregnancy outcomes. Eur J Obstet Gynecol Reprod Biol 2010; 150: 137-41.

34. Cavallasca JA, Laborde HA, Ruda-Vega H, Nasswetter GG. Maternal and fetal outcomes of 72 pregnancies in Argentine patients with systemic lupus erythematosus (SLE). Clin Rheumatol 2008; 27: 41-6.

35. Offenbacher S, Boggess KA, Murtha AP, Jared HL, Lieff S, Mckaig RG, et al. Progressive periodontal disease and risk of very preterm delivery. Obstet Gynecol 2006; 107: 29-36.

36. Ananth CV, Joseph KS, Oyelese Y, Demissie K, Vintzileos AM. Trends in preterm birth and perinatal mortality among singletons: United States, 1989 through 2000. Obstet Gynecol 2005; 105: 1084-91.

37. Huidobro A, Fulford A, Carrasco E. Incidencia de diabetes gestacional y su relación con obesidad en embarazadas chilenas. Rev Med Chile 2004; 132: 931-8.

38. Lanas F, Del Solar JA, Maldonado M, Guerrero M, Espinoza F. Prevalencia de factores de riesgo de enfermedad cardiovascular en una población de empleados chilenos. Rev Med Chile 2003; 131: 129-34.

39. Ananth CV, Vintzileos AM. Maternal-fetal conditions necessitating a medical intervention resulting in preterm birth. Am J Obstet Gynecol 2006 ;195: 1557-63. 